\title{
Los efectos de Prisionalización y su relación con el Trastorno Adaptativo ${ }^{1}$
}

\author{
Cajamarca Sarmiento, Jhon Edison²; Triana Barrios, Jhon Jairo3; \\ Jiménez-Jiménez, William Alejandro4.
}

\begin{abstract}
Resumen | La presente investigación tuvo como objetivo describir los efectos de prisionalización en internos condenados del complejo metropolitano de Bogotá y cuáles de estos inciden sobre el posible desarrollo de un trastorno adaptativo. La investigación de corte no experimental, tipo descriptivo longitudinal se llevó a cabo con 50 internos a quienes se les aplicó, en un primer momento, el instrumento IVIC (Instrumento para la Valoración Integral de Condenados); de estos, 15 personas fueron clasificadas con efectos de Prisionalización.
\end{abstract}

\section{Editado por}

William Alejandro Jiménez-Jiménez

Universidad de Boyacá, Colombia.

\section{Revisado por}

Carlos Elías Cifuentes Villalobos

Fundación Universitaria los

Libertadores
2Jhon Edison Cajamarca

Sarmiento $[\square]$

3 Jhon Jairo Triana Barrios

${ }^{4}$ William Alejandro Jiménez Jiménez
Psicólogo de la Fundación Universitaria Los Libertadores. Tesista de la presente investigación.

Psicólogo de la Fundación Universitaria Los Libertadores. Tesista de la presente investigación.

Asesor del trabajo de grado. Psicólogo de la Universidad Católica de Colombia. Magíster en Seguridad y Defensa Nacionales. Docente tiempo completo de la facultad de Psicología de la Fundación Universitaria los Libertadores. Contacto: wajimenezj@ libertadores.edu.co

\footnotetext{
${ }^{1}$ Artículo producto de la investigación del trabajo de grado con calificación aprobada que lleva el mismo título, presentado en la Facultad de Psicología de la Fundación Universitaria los Libertadores.
}

$\boldsymbol{\epsilon} \mid 54$ 
ascendencia 33.3\%, Responsabilidad 93.3\%, Estabilidad emocional 86.6\%, sociabilidad 20\%, Autoestima $33.3 \%$, Cautela $40 \%$, Originalidad $80 \%$, Relaciones Personales $33.3 \%$ y Vigor $66.6 \%$. Para futuras investigaciones se recomienda ampliar la muestra y hacer comparaciones significativas entre delitos y entre géneros para evaluar el Trastorno Adaptativo con los efectos de Prisionalización.

Palabras clave | Prisionalización, Trastorno Adaptativo, psicología penitenciaria.

\begin{abstract}
This research aimed to describe the effects of prisionalitation of condemned inmates in Bogota metropolitan complex and which of these affect the possible development of an adjustment disorder. The research was non experimental research, longitudinal descriptive conducted with 50 inmates who were applied, at first, the IVIC instrument (Instrumento para la Valoración Integral de Condenados); of these, 15 were classified as having effects of prisionalitation. Subsequently, to assess the presence of an adaptive disorder, this sample was applied to the P-IPG (Perfil-inventario de la Personalidad de Gordon), the results were: variables descent $33.3 \%$, Responsibility $93.3 \%$, Stability emotional $86.6 \%$, $20 \%$ sociability, self-esteem $33.3 \%$, Stealth $40 \%$ Originality $80 \%$, Personal Relations $33.3 \%$ and Vigor $66.6 \%$. For future research it is recommended to extend the sample and make meaningful comparisons between crimes and gender adjustment disorder to evaluate the effects of prisionalitation.
\end{abstract}

Key words | prisionalitation, adaptive disorder, correctional psychology

\title{
Introducción
}

Para el desarrollo de un estudio a profundidad de los efectos de Prisionalización con el Trastorno Adaptativo (TA) en ambientes carcelarios y penitenciarios, como uno de los tópicos de la psicología aplicada en contextos carcelarios, resultó ser un completo ejercicio de novedad y de interés científico, teniendo en cuenta las actuales circunstancias humanas y procedimentales en las cuales se encuentran las instituciones carcelarias dentro del contexto nacional. En los últimos años, gracias al interés de organismos gubernamentales, de entidades universitarias y de investigadores preocupados por el tema carcelario y penitenciario, se ha venido avanzando de una manera más fluida con aportes e investigaciones en el campo de la psicología jurídica aplicada a las instituciones totales. 
Este avance se debe al propio ejercicio práctico y a través de producción de las investigaciones en el contexto nacional (Díaz, 2011). Así, en el campo jurídico el psicólogo debe asumir una postura que beneficie, brinde herramientas y colabore en la toma de decisiones judiciales al Derecho. De acuerdo con lo afirmado por Soria (2005) (citado por Soria 2009), el psicólogo jurídico tiene en sus funciones cuatro roles fundamentales: Testigo, perito, testigo cualificado $y$ asesor.

De esta manera, según Morales y García (2010) dentro de las funciones del psicólogo jurídico en la práctica de su rol profesional están:

1. Evaluación y diagnóstico: este va direccionado a observar el comportamiento y condiciones psicológicas de los actores jurídicos.

2. Asesoramiento: orientar y guiar como perito psicológico a los órganos judiciales en conocimientos propios de su disciplina.

3. Intervención: generar un diseño para la prevención y tratamiento de los actores jurídicos, logrando una intervención tanto grupal como individual.

4. Formación y educación: entrenar y dar pautas a profesionales del sistema judicial en cuanto a técnicas psicológicas útiles para el desarrollo de una mejor intervención.

5. Campañas de prevención social ante la criminalidad: asesoramiento a campañas de información para las personas del común y que se encuentren en riesgo.

6. Investigación: estudio e investigación de la problemática de la psicología jurídica.

7. Victimologia: asesoramiento y colaboración a la situación de víctimas con el sistema legal.

8. Mediación: propiciar soluciones negociadas a los conflictos jurídicos a través de un proceso de mediación.

En cuanto al desarrollo y reconocimiento de la política penitenciaria en Colombia, fue alrededor de 1906 cuando se organizó y se dio origen al sistema de prisiones, años más tarde se

E $\mid 56$ 
fueron creando direcciones y divisiones que se encargaron de ejecutar las sanciones sociales de los individuos involucrados en situaciones legales (Gonzales, 2010). La necesidad de entender, y a la vez de estudiar, la conducta humana en ambientes totales no ha sido tan abordada (desde la evidencia empírica) a partir la Psicología, solamente se conoce el aporte de algunas áreas de interés como lo fue el desarrollado con la creación del Instrumento para la Valoración Integral de Condenados (IVIC) (Universidad Pontificia Javeriana, 2009). Así, uno de los efectos y consecuencias que se presentan en ambientes institucionalizados ha sido justamente el efecto de la Prisionalización como consecuencia de la pérdida de la libertad durante el tiempo establecido por la justicia.

El ingreso de un individuo a la prisión predispone desde su primer contacto un aislamiento social-afectivo que conlleva a la disminución de una serie de roles y factores sociales, familiares y sexuales, que a su vez, deteriora la autoestima e identidad (Ruiz et al, 2008). Cuando el proceso de incorporación al establecimiento ocurre, el individuo busca un lugar o grupo específico al cual pertenecer. El interno indaga sobre grupos con una identidad ya definida en cuanto a creencias, valores, normas de comportamiento o sistemas de comunicación, en donde se sienta aceptado y vinculado por los miembros del grupo (Sánchez; citado por Tapias, 2007).

De esta manera, la cárcel vista como sistema total cerrado que funciona con sus propias normas y de forma autónoma, resulta ser el lugar en el que el interno se ve sumergido en un cambio de contexto, debe asumir diversos roles y patrones de comportamiento así como nuevos códigos de comunicación y cambios en la economía, aunque es importante aclarar que el proceso de adaptación varía dependiendo cada individuo. Esto exige un esfuerzo adaptativo constante al encierro, que genera alteraciones a nivel emocional, afectivo, cognitivo y perceptivo (Echeverri, 2010).

Desde el momento en que inicia su condena en período cerrado e intramural por etapas considerablemente largas, el interno cambia todo su estilo de vida para lograr adaptarse a las diversas situaciones al interior de la prisión. Cuando estas alteraciones son superiores a la capacidad de adaptación del sujeto, se genera un fenómeno que Clemmer (citado por Pérez, 1991) denominó prisionización y Goffmann (por Pérez, 1991) enculturación, que básicamente es la progresiva adaptación por parte de la persona privada de la libertad a las nuevas habilidades y hábitos 
comportamentales. Esta situación se convierte en una lucha por la supervivencia y somete al interno a situaciones estresantes que dependen de factores personales, en donde las respuestas adaptativas de cada individuo juegan un papel determinante. Sin embargo, cuando el estrés es superior a los mecanismos de afrontamiento y de adaptación del individuo, se genera una sintomatología donde el estrés, la depresión y la ansiedad son las condiciones primarias para desarrollar los efectos por la Prisionalización (Echeverri, 2010).

Dentro de los temas de estudio de la Prisionalización se hace referencia al cambio en las cogniciones, la salud mental y física, pautas de comportamientos, entre otros factores, que una persona sufre al momento de ingresar a prisión. De igual forma, el hecho de enfrentar situaciones con alteración de la imagen corporal, exageración de las situación, alteraciones en la sexualidad y riesgo de suicidio en prisión (Ruiz citado por Tamara et al., 2011). La Prisionalización es una variable interviniente en la conducta del interno que afecta a casi todas las personas que ingresan a prisión con una intensidad diferente. Se establecen dos tipos de Prisionalización: la superficial y la definida. Por un lado, la Prisionalización superficial se distingue por el resultado de un proceso de adaptación normalizado y con una adaptación al ambiente de forma normal, del que no se podría definir como patológico. Por otro lado, la Prisionalización definida se identifica porque lleva al interno a mantener la dificultad para adaptarse al medio penitenciario, generando consecuencias patológicas más intensas y más extendidas en el tiempo (Echeverri, 2010).

\section{Variables que intervienen en la adaptación carcelaria}

La normal adaptación de un individuo al medio penitenciario está influenciada por diferentes variables que intervienen en este proceso. Estas variables, según Caballero (citado por Echeverri, 2010) son:

1. Edad: se puede entender como la acumulación de experiencias propias de la trayectoria de vida del interno, que facilitan la adaptación a la vida en prisión.

2. Nivel cultural: son todas las capacidades y conocimientos personales adquiridos por el interno, que le dan herramientas para la adaptación y un imaginario diferente de lo que será el cumplimiento de la condena. 
3. Trayectoria penal: hace referencia al interno que es reincidente, quien tendrá menos efectos al ingresar por segunda vez a la prisión.

Dentro del contexto colombiano, El Instituto Nacional Penitenciario y Carcelario (INPEC) cuenta con un instrumento psicométrico para definir variables de riesgo del interno, entre ellas el efecto de Prisionalización. Por medio del instrumento psicométrico llamado Diseño, Validación e implementación de Instrumentos científicos para el proceso de valoración, clasificación y seguimiento en el tratamiento penitenciario de la población condenada en los establecimientos de reclusión de orden nacional - IVIC, el consejo de evaluación y tratamiento (CET) del centro carcelario, se encarga de observar, cuantificar y analizar las necesidades de cada interno. Esto permitió conocer la realidad y los riesgos del personal condenado. $Y$ a partir de lo anterior se ha creado un equipo interdisciplinario de 8 áreas (Seguridad y Custodia, Antropología, Psicología, Derecho jurídico, Terapia Ocupacional, Trabajo Social y Educación) que son los encargados de velar por la resocialización y satisfactoria adaptación del interno al medio penitenciario (Universidad Pontificia Bolivariana, 2009).

Tabla 1 / Variables efectos de Prisionalización

\begin{tabular}{cccc}
\hline \hline VARIABLES & DIMENSIONES & SUB-DIMENSIÓN & INDICADORES \\
\hline \hline Riesgo psicopatológico & Factores & $\begin{array}{c}\text { Riesgo cognitivo } \\
\text { personalidad } \\
\text { predisponente }\end{array}$ & $\begin{array}{c}\text { Orientación } \\
\text { Crisis emocional } \\
\text { sustancias }\end{array}$ \\
psicológicas & Recursos cognitivos & Ansiedad & Estrés \\
& & Depresion & $\begin{array}{c}\text { Locus de control } \\
\text { Indiferencia a la } \\
\text { norma }\end{array}$ \\
\hline
\end{tabular}




\begin{tabular}{|c|c|c|c|}
\hline VARIABLES & DIMENSIONES & SUB-DIMENSIÓN & INDICADORES \\
\hline $\begin{array}{c}\text { Comportamiento } \\
\text { social }\end{array}$ & & $\begin{array}{l}\text { Perspectiva del otro } \\
\text { Relaciones } \\
\text { interpersonales } \\
\text { Apoyo social }\end{array}$ & $\begin{array}{c}\text { Habilidades de } \\
\text { comunicacion } \\
\text { Habilidades de } \\
\text { oposición } \\
\text { Apoyo social } \\
\text { emocional }\end{array}$ \\
\hline
\end{tabular}

Fuente: Instrumento IVIC - Universidad Pontificia Bolivariana (2009).

A pesar del aporte presentado previamente, el estudio científico de los efectos de Prisionalización en el interno ha mostrado una importancia muy poca en su análisis, curso, prevalencia y alternativas de tratamiento. Para comprender la importancia del estudio de este fenómeno, cabe resaltar las investigaciones y reflexiones realizadas por Pérez-Fernández y Redondo-Illescas (1991), Valdez (2009); Joya y Quintero (2011); Altamirano y Cuevas (2012); Mena y Rueda (2012); Escaff-Silva, Feliú-Vergara, Estévez-Merello, Torrealba-Henríquez (2013); Carreño (2013); Medina, Osorno, Reyes, Ríos y Jiménez (2014); y Martínez (2014).

\section{Definición de Trastorno Adaptativo (TA)}

A partir de lo anteriormente mencionado, en donde se contextualiza la evaluación del efecto de la Prisionalización y su desarrollo en las cárceles y penitenciarías colombianas, es menester mencionar la conceptualización del trastorno adaptativo que resulta ser de suma importancia para comprender con mayor profundidad los efectos psicológicos del encarcelamiento.

Según el DSM IV-TR (2002), el Trastorno Adaptativo (TA) es la aparición de respuestas emocionales o de tipo comportamental ante la presencia de algún factor psicosocial claramente identificado. Así, la sintomatología del trastorno adaptativo debe estar presente después de tres (3) meses del evento estresante. De acuerdo con este manual de diagnóstico, existen varios subtipos de Trastorno Adaptativo: 1. TA con estado de ánimo depresivo; 2. TA con ansiedad; 3. TA mixto, con ansiedad y estado de ánimo depresivo; 4. TA con trastorno de comportamiento; 5. TA con alteración mixta de las emociones y el comportamiento; y 6 . TA no especificado.

$\boldsymbol{\epsilon} \| 60$ 
Los diagnósticos según el DSM IV-TR (2002) para trastornos adaptativos varían según la exposición del individuo a un evento estresante en específico. Con esto se puede explicar que los síntomas comportamentales y emocionales dependen de un evento estresante psicosocial ya identificado. El diagnóstico de Trastorno Adaptativo no se aplica cuando los síntomas representan una reacción de duelo. Dichos síntomas deben estar presentes durante los siguientes tres (3) meses al inicio de la presencia del estresor. Es ahí donde se ve la expresión clínica del trastorno como tal, en donde se ve afectada la actividad social y profesional del individuo.

Por otro lado, de acuerdo con la clasificación Internacional de las Enfermedades (CIE 10, OMS 1992) los TA son definidos como estados de malestar subjetivo acompañado de alteraciones emocionales que, por lo general interfieren con la actividad social y que aparecen en el período de adaptación a un cambio biográfico o a un acontecimiento vital estresante. Son dos definiciones que en su contexto y origen son completamente diferentes, pero ambas tienen un factor común denominador: afectan el buen desenvolvimiento social del sujeto afectado por el factor estresante.

A partir de esta contextualización operacional de los dos grandes manuales de clasificación de las enfermedades (DSM y CIE), existen otras definiciones propuestas por diversos autores que se aproximan al conocimiento operacional de tipo psicológico del TA. Por ejemplo, la Entidad Colombiana de Salud (2009) define el TA como un tipo de trastorno de ajuste acompañado de malestar emocional y comportamental, con un alto significado clínico en respuesta al estrés psicosocial identificable en un período después de tres meses de la aparición del evento estresor.

Después de ahondar en lo referente al TA, el modelo explicativo cognitivo creado por Botella, Baños y Guillen (citado por Andreu, 2011), explica que si una persona se enfrenta a una situación estresante, interpreta dicha situación como altamente amenazante, y además de esto tiene la percepción de que no cuenta con los suficientes recursos para asimilarla y enfrentarla, creará una evaluación negativa cargada de sentimientos y pensamientos de carácter amenazantes y negativos. Esto a su vez genera alteraciones emocionales, de ansiedad, depresión y miedo, asociadas con alteraciones en el comportamiento. Es por esto que este modelo se centra en la importancia que tiene la evaluación negativa (tanto del acontecimiento como de las capacidades propias de afrontamiento de la situación), en la generación de un trastorno adaptativo. 


\section{Evaluación de los Trastornos Adaptativos}

En la actualidad es muy poca la información empírica que existe del estudio de los trastornos adaptativos en ambientes totales. Normalmente se encuentran investigaciones realizadas en el campo de la salud y en la psicología clínica, pero pocas en la psicología penitenciaria. Por ejemplo, se pueden destacar los aportes en el campo de la psicología clínica y de la salud de Gómez, Aldana, Carreño y Sánchez (2006); Fernández (2008); Murillo, Salcedo y Bohórquez (2009); Herrero y Cano (2010); Ojeda (2010); Andreu (2011); Zeas, Coello, De las Torres e Iglesias (2012); en el ámbito laboral como la investigación desarrollada por Mingote, Gálvez, Cuadrado y Gutiérrez (2009); en el ámbito de sociocultural, el aporte de Zarza y Sobrino (2007); y en el ambiente penitenciario, la investigación desarrollada por Ruiz (2008).

A propósito de la importancia de la evaluación psicológica, el Trastorno Adaptativo es uno de los trastornos menos estudiados debido al poco interés que despierta en científicos e investigadores (Molés et al, 2012). Debido a esto, los instrumentos de diagnóstico conocidos para la evaluación psicológica del Trastorno Adaptativo son muy escasos hasta el momento. Por ende, para los efectos de esta investigación se decidió utilizar el P-IPG (Perfil-Inventario de la Personalidad de Gordon), que mide factores relacionados con adaptación, así como aspectos de ascendencia, responsabilidad, estabilidad emocional, sociabilidad, relaciones personales y vigor.

\section{Método}

\section{Tipo de investigación}

Esta investigación fue no experimental, tipo descriptivo y corte longitudinal. (Hernández, Fernández \& Baptista, 2006).

\section{Muestra}

Corresponde a una muestra no probabilística de tipo intencional, conformada por internos condenados del Complejo Metropolitano de Bogotá (PICOTA) incluidos en la fase de observación y diagnóstico, los cuales fueron remitidos por el CET (Consejo de Evaluación y Tratamiento) para

€ $\| 62$ 
ser clasificados en la fase de Alta. Conformada de población interna donde se tomó una muestra representativa originalmente de 50 internos, de los cuales se seleccionaron 15, a quienes se les aplicó el instrumento IVIC para determinar cuáles de estos presentan efectos de Prisionalización. Tres meses después se aplicó la prueba P-IPG con el fin de observar cuáles internos presentan Trastorno Adaptativo.

\section{Instrumentos}

\section{Perfil inventario de la personalidad (P-IPG)}

Esta prueba combina dos instrumentos, el inventario de personalidad de Gordon y el perfil de personalidad de Gordon; anteriormente las dos se manejaban por separado, pero en 1978 se combinaron para crear el P-IPG. El objetivo de esta prueba es medir rasgos estables de la personalidad y un factor de autoestima relacionados con la adaptación y eficiencia del sujeto en situaciones sociales educativas y laborales. Esta prueba mide cuatro rasgos de la personalidad significativos en el funcionamiento diario del individuo normal: 1. ascendencia (A) la cual hace referencia a la forma en que el interno toma de decisiones y su posición frente a situaciones que requieren de liderazgo, $\mathbf{2}$. Responsabilidad (R) que se relaciona con el ámbito laboral, la tenacidad y responsabilidad frente a situaciones de la vida diaria; 3. Estabilidad emocional (E) se refiere a la estabilidad emocional del sujeto, la ansiedad y tensión nerviosa, tolerancia a la frustración; 4. Sociabilidad (S) este ítem se refiere a las relaciones sociales y la posición del interno frente a trabajos en equipo.

Adicionalmente, el inventario mide otros cuatro rasgos: cautela, originalidad relaciones personales y vigor, que combinados proporcionan una apreciación importante de la personalidad y una medida de autoestima ( $A E$ ) basada en la sumatoria del perfil de personalidad de Gordon, donde se tienen en cuenta los rasgos de adaptación.

\section{IVIC. Instrumento para la Valoración Integral de Condenados}

Instrumento científico creado tras el convenio 1310 del 2009 con la Universidad Pontificia Bolivariana de Bucaramanga, con el fin de hacer más objetivo el proceso de clasificación de fase de seguridad, tratamiento penitenciario y seguimiento de los internos condenados en las cárceles 
del país. Dicho instrumento cuenta con variables y factores de diferentes áreas, tanto de salud como de seguridad. Se trata de un trabajo interdisciplinar dirigido al estudio y valoración física y psicosocial de los sujetos privados de su libertad.

EI IVIC es una prueba psicométrica que consta de 164 ítems con múltiples respuestas, que abordan aspectos y variables tales como la orientación, espacialidad, tiempo, peligrosidad, reincidencia, pautas de crianza, relaciones interpersonales, entre otras, en internos pertenecientes a la fase de observación y diagnóstico. EI IVIC como instrumento de medición no pretende suplantar las funciones del profesional, por el contrario es una guía para facilitar la identificación de factores de riesgo del interno condenado.

\section{Procedimiento}

La presente investigación se realizó siguiendo un proceso con un total de 11 fases descritas a continuación: 1 . Se seleccionó una muestra por conveniencia de 50 internos pertenecientes a la fase de observación y diagnóstico del Complejo Metropolitano de Bogotá (PICOTA); 2. Luego de escogida esta muestra fue aplicado el instrumento del IVIC para la identificación de los internos con mayor incidencia en los efectos de prisionalización; 3. Se procedió a la sistematización de los resultados por medio del software SISIPECWEB para identificar las variables más altas de los efectos de prisionalización; 4. Luego de haber llevado la fase de la aplicación del IVIC y de haber identificado a los internos con efectos de Prisionalización, se obtuvo una muestra de estudio de 15 internos que presentaron dichos efectos. Con estos resultados se obtuvo el grupo de estudio con el cual se trabajó durante toda la investigación; 5 . Se procedió a esperar un tiempo promedio de 2 a 3 meses; 6 . Se realizó la aplicación de la prueba P-IPG; 7. Se realizó la calificación para definir las variables relacionadas con Trastorno Adaptativo; 8. Se interpretaron los resultados arrojados de ambas pruebas; 9. Hizo la correlación de los efectos de Prisionalización con el Trastorno Adaptativo: 10. Con estas interpretaciones, se analizaron los resultados finales; 11 . Y finalmente se discutieron los resultados y se establecieron las conclusiones de la investigación. 


\section{Resultados}

Tras la aplicación del instrumento IVIC a la muestra total de 50 internos pertenecientes a la fase de observación y diagnóstico, se logró identificar como primer análisis que el $30 \%$ de ellos presentaron efectos de Prisionalización (15/50). Una vez realizado el filtro se procedió a trabajar con esta muestra total de 15 sujetos privados de su libertad. De acuerdo con los resultados obtenidos con el IVIC para el análisis de los resultados, estos se distribuyeron según las variables del instrumento de la siguiente manera:

Tabla 2 | Resultados variables efectos de prisionalización IVIC

La figura 1 muestra la representación total de variables del IVIC en porcentajes de la siguiente manera:

\begin{tabular}{|c|c|c|c|c|c|c|c|c|c|c|c|c|c|c|c|}
\hline VARIABLES IVIC & 1 & 2 & 3 & 4 & 5 & 6 & 7 & 8 & 9 & 10 & 11 & 12 & 13 & 14 & 15 \\
\hline $\begin{array}{l}\text { Riesgo } \\
\text { psicopatológico }\end{array}$ & Bajo & Medio & Medio & Bajo & Bajo & Medio & Bajo & Bajo & Medio & Medio & Bajo & Bajo & Medio & Medio & Medio \\
\hline $\begin{array}{l}\text { Condiciones } \\
\text { psicológicas }\end{array}$ & Medio & Bajo & Bajo & Medio & Medio & Bajo & Medio & Bajo & Medio & Bajo & Bajo & Medio & Medio & Bajo & Bajo \\
\hline $\begin{array}{l}\text { Comportamiento } \\
\text { social }\end{array}$ & Medio & Bajo & Bajo & Bajo & Bajo & Bajo & Bajo & Bajo & Medio & Medio & Bajo & Bajo & Bajo & Bajo & Bajo \\
\hline $\begin{array}{l}\text { Factores } \\
\text { predisponentes }\end{array}$ & Bajo & Bajo & Bajo & Bajo & Bajo & Bajo & Bajo & Bajo & Medio & Medio & Bajo & Bajo & Bajo & Bajo & Bajo \\
\hline $\begin{array}{l}\text { Recursos } \\
\text { cognitivos }\end{array}$ & Medio & Medio & Bajo & Medio & Alto & Bajo & Bajo & Bajo & Bajo & Bajo & Medio & Medio & Bajo & Medio & Bajo \\
\hline Riesgo cognitivo & Medio & Bajo & Bajo & Bajo & Bajo & Bajo & Bajo & Bajo & Bajo & Bajo & Bajo & Medio & Bajo & Bajo & Bajo \\
\hline Personalidad & Medio & Alto & Medio & Medio & Bajo & Medio & Bajo & Bajo & Bajo & Medio & Medio & Bajo & Medio & Medio & Medio \\
\hline $\begin{array}{l}\text { Consumo de } \\
\text { sustancias }\end{array}$ & Alto & Bajo & Alto & Alto & Medio & Alto & Alto & Medio & Alto & Alto & Medio & Alto & Medio & Alto & Alto \\
\hline Ansiedad & Alto & Bajo & Alto & Alto & Medio & Medio & Alto & Medio & Alto & Bajo & Bajo & Alto & Alto & Bajo & Bajo \\
\hline Estrés & Alto & Bajo & Bajo & Bajo & Medio & Medio & Alto & Bajo & Bajo & Alto & Bajo & Alto & Alto & Bajo & Bajo \\
\hline Depresión & Bajo & Bajo & Medio & Alto & Medio & Medio & Medio & Bajo & Alto & Alto & Medio & Medio & Medio & Medio & Bajo \\
\hline Perspectiva otro & Alto & Bajo & Bajo & Bajo & Bajo & Medio & Bajo & Bajo & Bajo & Medio & Bajo & Bajo & Alto & Bajo & Bajo \\
\hline $\begin{array}{l}\text { Relaciones } \\
\text { interpersonales }\end{array}$ & Alto & Medio & Bajo & Medio & Alto & Alto & Medio & Bajo & Alto & Bajo & Bajo & Bajo & Bajo & Medio & Medio \\
\hline Apoyo social & Alto & Bajo & Alto & Bajo & Bajo & Bajo & Bajo & Alto & Alto & Alto & Bajo & Bajo & Bajo & Bajo & Bajo \\
\hline Orientación & Medio & Bajo & Bajo & Bajo & Bajo & Bajo & Bajo & Bajo & Bajo & Medio & Bajo & Medio & Bajo & Bajo & Bajo \\
\hline Crisis emocional & Bajo & Bajo & Bajo & Medio & Bajo & Bajo & Bajo & Bajo & Bajo & Bajo & Bajo & Bajo & Bajo & Bajo & Bajo \\
\hline
\end{tabular}




\begin{tabular}{|c|c|c|c|c|c|c|c|c|c|c|c|c|c|c|c|}
\hline VARIABLES IVIC & 1 & 2 & 3 & 4 & 5 & 6 & 7 & 8 & 9 & 10 & 11 & 12 & 13 & 14 & 15 \\
\hline Locus de control & Bajo & Alto & Bajo & Bajo & Bajo & Bajo & Bajo & Bajo & Bajo & Bajo & Bajo & Alto & Bajo & Bajo & Bajo \\
\hline Indiferencia norma & Bajo & Bajo & Alto & Bajo & Medio & Medio & Medio & Bajo & Alto & Bajo & Medio & Medio & Alto & Bajo & Alto \\
\hline $\begin{array}{l}\text { Habilidades } \\
\text { comunicación }\end{array}$ & Alto & Bajo & Medio & Bajo & Alto & Bajo & Bajo & Bajo & Alto & Bajo & Alto & Alto & bajo & Medio & Medio \\
\hline $\begin{array}{l}\text { Habilidades } \\
\text { oposición }\end{array}$ & Bajo & Bajo & Alto & Bajo & Bajo & Bajo & Alto & Alto & Alto & Alto & Bajo & Alto & Bajo & Bajo & Bajo \\
\hline $\begin{array}{l}\text { Apoyo social } \\
\text { emocional }\end{array}$ & Alto & Bajo & Alto & Bajo & Bajo & Bajo & Medio & Alto & Alto & Alto & Bajo & Alto & Bajo & Bajo & Bajo \\
\hline
\end{tabular}

Riesgo psicopatológico 53.3\% (8/15), condiciones psicológicas 46.6 (7/15), comportamiento social $20 \%$ (3/15), factores predisponentes $13.3 \%$ (2/15), recursos cognitivos $46.6 \%(7 / 15)$, riesgo cognitivo $13.3 \%(2 / 15)$, personalidad $66.6 \%$ (10/15), consumo de sustancias $93.3 \%(14 / 15)$, ansiedad 66.6\% (10/15), estrés $46.6 \%(7 / 15)$, depresión $73.3 \%(11 / 15)$, perspectiva del otro $26.6 \%(4 / 15)$, relaciones interpersonales $60 \%$ (9/15), apoyo social 33.3\% (5/15), orientación $20 \%(3 / 15)$, crisis emocional $6.6 \%(1 / 15)$, afectividad $66.6 \%(10 / 15)$, locus de control $13.3 \%$ $(2 / 15)$, indiferencia a la norma $60 \%(9 / 15)$, habilidades de comunicación $13.3 \%$ (7/15), habilidades de oposición 33.3\% (5/15), apoyo social emocional 40\% (6/15).

Figura 1 / Representación del total de variables IVIC en porcentajes.

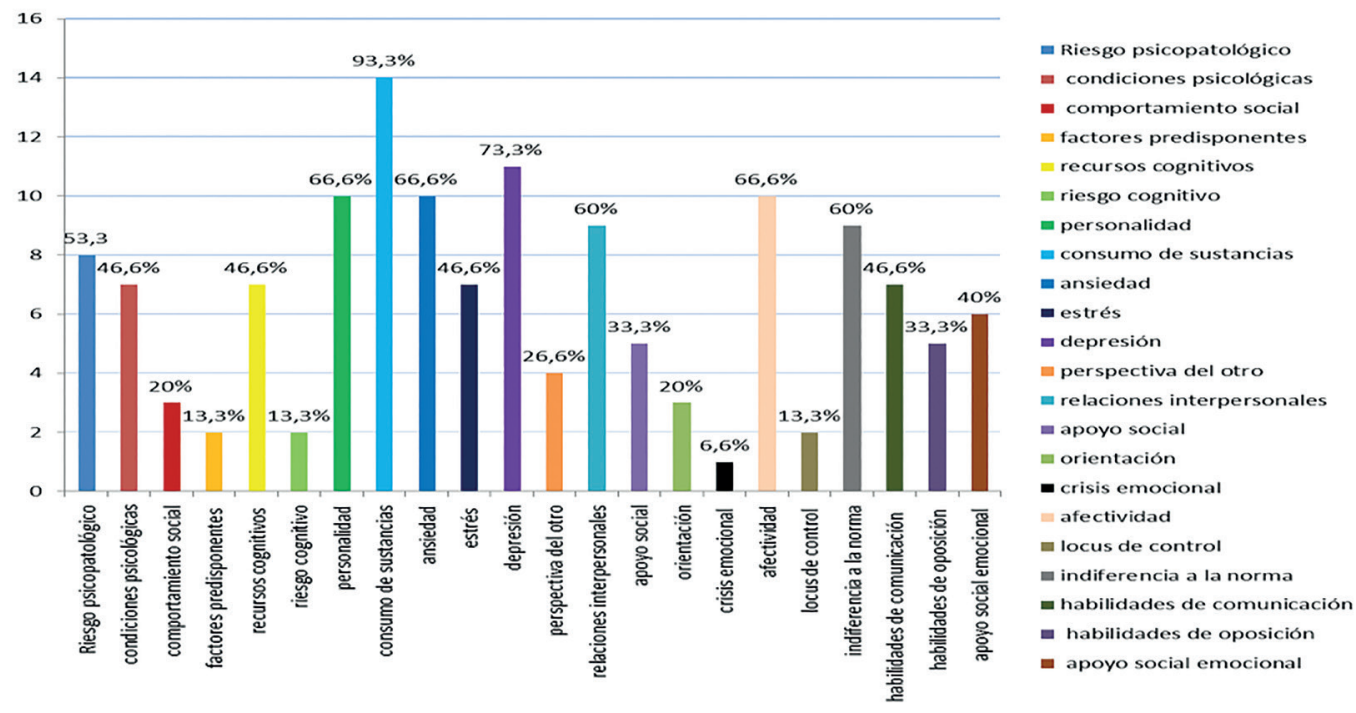

$\boldsymbol{\varepsilon} \| 66$ 
En la figura 2 se describen los puntajes obtenidos por cada interno con un total de 22 variables de prisionalización. Se tienen en cuenta las variables que puntuaron medio y alto, en el resultado del instrumento. Sujeto 1, 63.3\% (14/22); sujeto 2, 27.2\% (6/22); sujeto 3, 45.5\% (10/22); sujeto 4, $40.9 \%$ (9/22); sujeto 5, 40.9\% (9/22); sujeto 6, 45.5\% (10/22); sujeto 7, 45.5\% (10/22); sujeto 8 , $27.2 \%$ (5/22); sujeto 9, 59\%(13/22); sujeto $10,59 \%$ (13/22); sujeto $11,31.8 \%(7 / 22)$; sujeto 12 , $45.4 \%$ (10/22); sujeto $13,54.4 \%$ (10/22); sujeto $14,36.3 \%$ (8/22); sujeto $15,31.8(7 / 22)$.

Figura 2 / Representación total de sujetos por total de variables
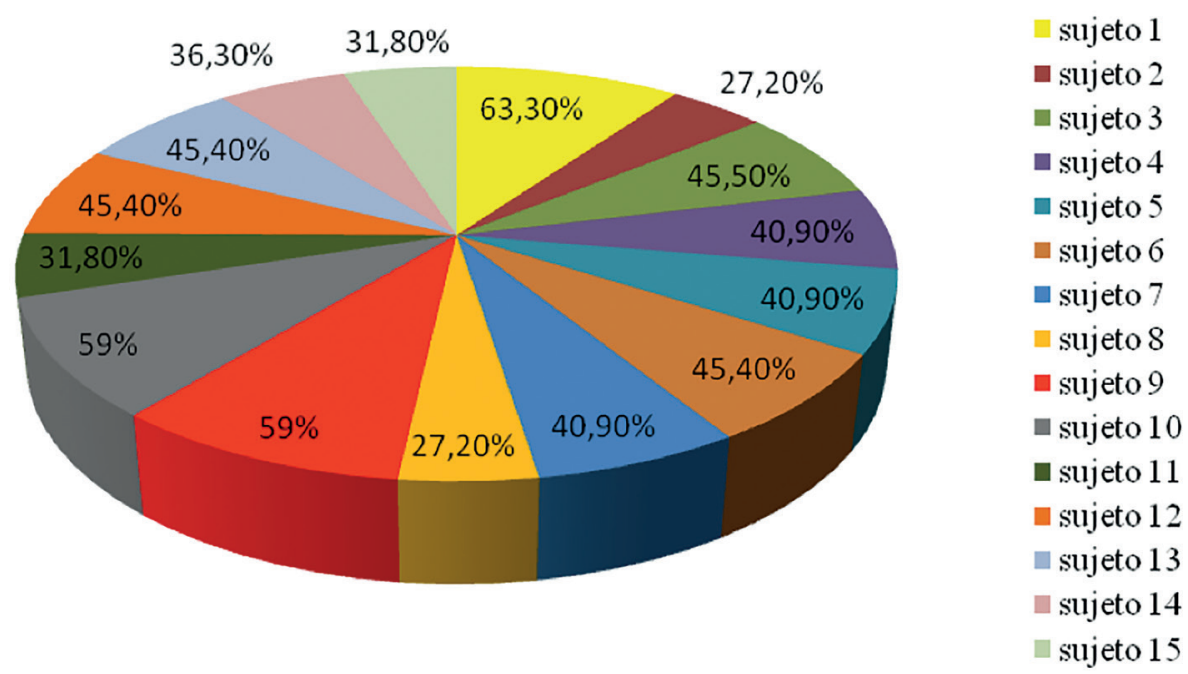

\section{P-IPG}

Tras la aplicación del instrumento a la muestra ya filtrada de 15 internos pertenecientes a la fase de observación y diagnóstico, se obtienen los siguientes resultados. 
Tabla3 / Resultados instrumento P-IPG para Trastorno Adaptativo

\begin{tabular}{|c|c|c|c|c|c|c|c|c|c|}
\hline \multirow[b]{2}{*}{ SUJETOS } & \multicolumn{5}{|c|}{ PPG } & \multicolumn{4}{|c|}{ IPG } \\
\hline & A & $\mathrm{R}$ & E & $S$ & $A E$ & C & 0 & $P$ & V \\
\hline 1 & Alto & Bajo & Bajo & Media & Media & Alta & Baja & Media & Alta \\
\hline 2 & Medio & Bajo & Media & Alta & Media & Alta & Baja & Alta & Alta \\
\hline 3 & Bajo & Bajo & Bajo & Media & Baja & Baja & Baja & Baja & Baja \\
\hline 4 & Alto & Bajo & Bajo & Media & Baja & Baja & Baja & Baja & Baja \\
\hline 5 & Medio & Bajo & Bajo & Alta & Media & Media & Baja & Media & Baja \\
\hline 6 & Alto & Bajo & Bajo & Media & Media & Media & Baja & Media & Baja \\
\hline 7 & Medio & Bajo & Bajo & Bajo & Media & Baja & Baja & Baja & Baja \\
\hline 8 & Alto & Bajo & Bajo & Alta & Media & Alta & Baja & Alta & Alta \\
\hline 9 & Bajo & Bajo & Alta & Bajo & Media & Alta & Media & Alta & Baja \\
\hline 10 & Alto & Bajo & Bajo & Alta & Media & Baja & Baja & Media & Baja \\
\hline 11 & Medio & Media & Bajo & Alta & Media & Alta & Alta & Alta & Alta \\
\hline 12 & Bajo & Bajo & Bajo & Media & Media & Baja & Baja & Baja & Baja \\
\hline 13 & Alto & Bajo & Bajo & Media & Baja & Alta & Alta & Media & Baja \\
\hline 14 & Bajo & Bajo & Bajo & Media & Baja & Baja & Baja & Baja & Baja \\
\hline 15 & Bajo & Bajo & Bajo & Bajo & Baja & Alta & Baja & Alta & Media \\
\hline
\end{tabular}

En la figura 3 se muestra la representación total de variables del P-IPG en porcentajes de la siguiente manera. Ascendencia 33.3\% (5/15), Responsabilidad 93.3\% (14/15), Estabilidad emocional 86.6\% (13/15), Sociabilidad 20\% (3/15), Autoestima 33.3\% (5/15), Cautela 40\% (6/15), Originalidad $80 \%$ (12/15), Relaciones personales $33.3 \%$ (5/15) y Vigor $66.6 \%(10 / 15)$. 
Figura 3 / Representación del total de variables P-IPG en porcentajes

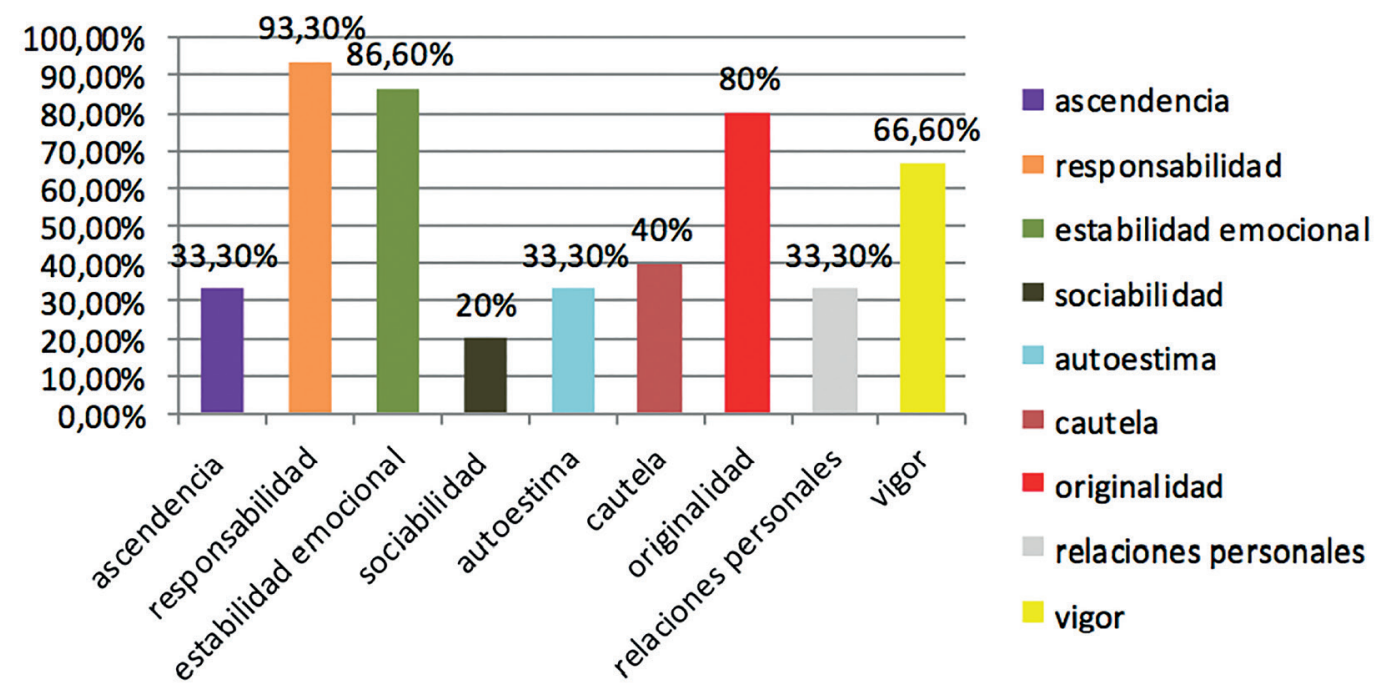

En la figura 4 se describen los puntajes obtenidos por cada interno de un total de 9 variables de la prueba P-IPG, se tienen en cuenta las variables que puntuaron bajo en el resultado del instrumento. Sujeto 1. 33.3\% (3/9); sujeto 2, 22.2\% (1/9); sujeto 3, 88.8\% (8/9); sujeto $4,77.7 \%$ (7/9); sujeto 5, 44.4\% (4/9); sujeto 6, 44.4\% (4/9); sujeto 7, 77.7\% (7/9); sujeto 8, $33.3 \%$ (2/9); sujeto 9, $44.4 \%$ (4/9); sujeto $10,55.5 \%$ (5/9); sujeto $11,11.1 \%$ (1/9); sujeto $12,77.7 \%$ (7/9); sujeto $13,44.4 \%$ (4/9); sujeto $14,88.8 \%$ (8/9); sujeto $15,66.6 \%$ (6/9). 
Figura 4 / Representación del total de sujetos por total de variables TA

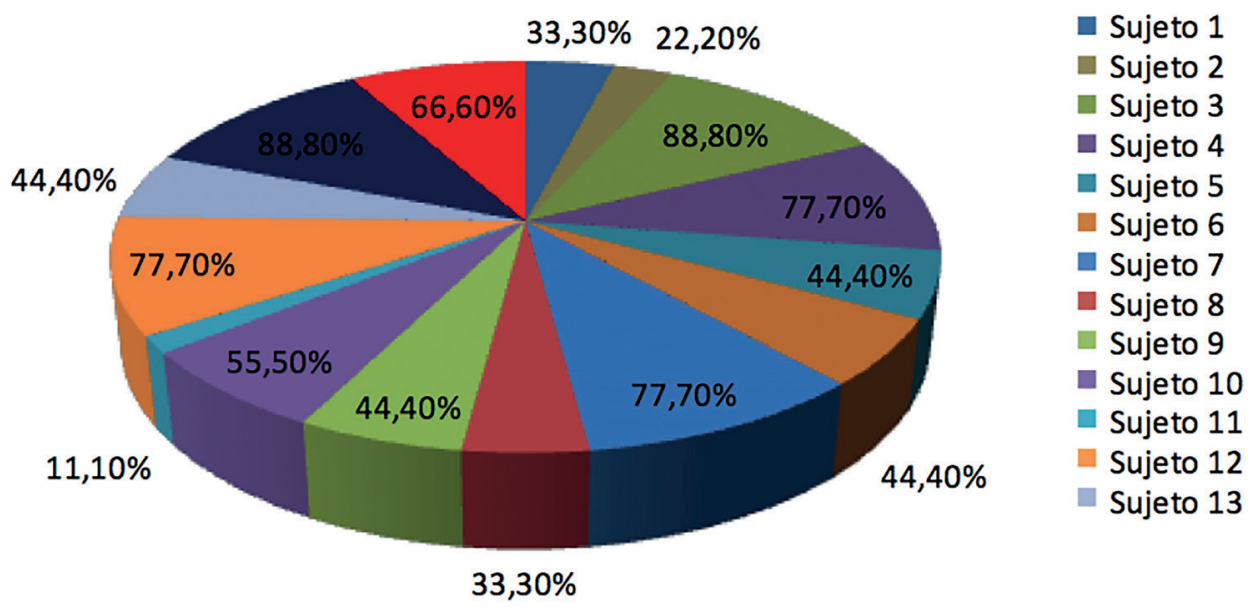

A partir de la aplicación de los instrumentos (IVIC y P-IPG) con la finalidad de conocer cuáles fueron los efectos de Prisionalización que incidieron en el desarrollo de un Trastorno Adaptativo, se procedió a analizar los resultados de forma general teniendo en cuenta las variables de Prisionalización predominantes, para contextualizarlas con las variables más sobresalientes de inadaptación de P-IPG.

En primer lugar, tras desarrollar el análisis de los resultados expuestos en la figura 1 y figura 3 de variables generales, tanto de efectos de Prisionalización (IVIC) y Trastornos Adaptativos (PIPG), se observó que como variables predominantes de los efectos de prisionalización se tomó como referencia de corte el 50\%, para determinar cuáles son las más influyentes.

En cuanto al instrumento IVIC, la variable con mayor puntuación es la de consumo de sustancias con un total de $98.3 \%$, es decir se encuentra presente en 14 de los 15 internos de la población, un aspecto destacado en el análisis de resultados. Le sigue las variables de depresión con un $73.3 \%$ y las variables de personalidad y estrés, cada una con un $66.6 \%$ en la población total; y finalmente la presencia en un porcentaje alto de la variable de riesgo psicopatológico,

ᄐ 170 
con un $53.3 \%$ en la totalidad del análisis. Revisando este resultado se puede evidenciar la relación que tienen estas variables de Prisionalización, ya que el consumo de sustancias puede desencadenar altos niveles de ansiedad y depresión, que además pueden generar un desgaste emocional, alteraciones en el estado de ánimo y cambios comportamentales. Sumado todo esto sin un tratamiento adecuado, puede generarle al interno un riesgo psicopatológico (Trastorno Adaptativo) (Ver figura 1).

Por otro lado, en el análisis general de variables de la prueba P-IPG arroja que la variable con más presencia entre los internos es la de Responsabilidad con 93.3\%, con presencia en 14 de los 15 sujetos que se tomaron como muestra final, lo cual genera una inestabilidad en las labores diarias; seguido por la variable de Estabilidad emocional con un $86.6 \%$, que afecta de manera directa al sujeto con altos niveles de ansiedad y baja tolerancia a la frustración; en cuanto a Originalidad, se observó un $80 \%$, lo que genera dificultad al momento de enfrentarse a labores de alta dificultad; por último se encuentra la variable de Vigor, que mantiene un puntaje total de $66.6 \%$, que altera la vitalidad y el entusiasmo del interno en sus labores del diario vivir (Ver figura 3).

Analizando lo anterior se puede evidenciar cómo las variables de efectos de Prisionalización inciden directamente en la manifestación del Trastorno Adaptativo, ya que el consumo de sustancias puede generar alteraciones emocionales que afectan la vitalidad al realizar las actividades correspondientes, llevando al sujeto a perder el compromiso con las mismas.

En cuanto a las puntuaciones de los resultados del IVIC para variables generales por sujeto, se evidencia que los que presentan mayor cantidad de variables de efectos de Prisionalización son los sujetos 1- 9 y 10; el primero obtiene un porcentaje de $63.3 \%$ que equivale a 14 de 22 variables y por último, los sujetos 9 y 10 con un porcentaje de $59 \%$, equivalente a 13 de 22 variables de efectos de Prisionalización. (Ver figura 2).

Por otro lado, los resultados obtenidos del P-IPG en cuanto a variables generales por sujeto, evidencian que los internos que presentan mayor número de variables de Trastorno Adaptativo son los sujetos 3 y 14 de la muestra general, cada uno obteniendo un porcentaje del $88.8 \%$ que equivale a 8 de 9 variables. Seguido de ellos están los sujetos 4,7 y 12 que puntúan $77.7 \%$, equivalente a 7 de 9 variables. Luego se encuentra el sujeto 15 con un porcentaje de $66.6 \%$, 
que equivale a 6 de 9 variables y por último el sujeto 10 con una puntuación de $55.5 \%$ equivalente a 5 de 9 variables de Trastorno Adaptativo. (Ver figura 4).

En cuanto a los resultados anteriores se puede evidenciar que solo uno de los sujetos obtuvo puntuación alta en cuanto a efectos de Prisionalización y Trastorno Adaptativo.

\section{Interpretaciones individuales}

\section{sujeto1}

En el sujeto 1 se pudieron evidenciar variables altas en efectos de Prisionalización como el consumo de sustancias, estrés y depresión, que pueden desencadenar baja responsabilidad e interés en las actividades diarias, de igual forma se evidenciaron dificultades para adaptarse al contexto penitenciario. Por otro lado, las puntuaciones altas en ámbitos relacionados con lo emocional en la prueba IVIC, como lo son las habilidades sociales, perspectiva del otro y apoyo emocional, como se evidencia en la figura 3, son variables que al permanecer estables en el tiempo, hacen parte de los criterios para el desarrollo de un Trastorno Adaptativo, como se evidencia en la figura 20, en la que se puede apreciar la baja puntuación en variables como Estabilidad emocional (E), que evidencia la presencia de excesiva ansiedad y desinterés en relacionarse con otros internos (Originalidad), debido a la alteración en las habilidades sociales.

\section{Sujeto 2}

Tras la calificación de los resultados, se puede interpretar que la puntuación referente a la variable de locus de control relacionada con los efectos de Prisionalización en el contexto de la pérdida de interés y de responsabilidad en el interno, tiene un nivel alto; lo que podría incidir en el desarrollo de un Trastorno Adaptativo, así como se evidencia en la variable "Responsabilidad" 


\section{Sujeto 3}

Analizando las variables altas (IVIC) y variables bajas (P-IPG) correspondientes al sujeto 3, se llega a la conclusión de que se alteran por los efectos de Prisionalización en las áreas: emocional, social y relaciones interpersonales. Un puntaje elevado en consumo de sustancias psicoactivas puede tener como antecedente alteraciones emocionales y problemas de estrés y depresión, relacionado con los primeros meses de estancia en la prisión, esto sin un tratamiento adecuado que brinde herramientas al interno para sobrellevar la situación, puede desencadenar posteriormente un Trastorno Adaptativo, en donde las condiciones psicológicas y los recursos cognitivos afectarán de manera más profunda, áreas como la responsabilidad, relacionada con pérdida de entusiasmo en las labores diarias, alteraciones emocionales y pérdida de la autoestima. Factores que están directamente relacionados con los Trastornos de Adaptación.

\section{Sujeto 4}

La estabilidad emocional, uno de los factores que se ve alterado por el desarrollo de un Trastorno Adaptativo, puede estar relacionado con la ansiedad y la depresión de los primeros meses de estancia en la prisión, como es evidente tras el análisis de los resultados del sujeto 4 . De la misma manera, la evidencia de puntuaciones bajas en autoestima y relaciones personales, puede tener como predisponente el hecho de tener puntuaciones altas en los primeros meses de prisión en afectividad y crisis emocionales, las cuales generan baja tolerancia a la frustración y lo llevan a tomar decisiones apresuradas respecto a su vida diaria, generando consecuencias en su entorno social y en sus relaciones interpersonales.

\section{Sujeto 5}

Los recursos cognitivos del interno no son suficientes para afrontar la situación en prisión, lo que hace que los niveles de ansiedad y depresión sean elevados. La variable de consumo de sustancias se encuentra presente, probablemente para disminuir los niveles de ansiedad. Durante los primeros meses en prisión se vieron afectadas las relaciones interpersonales y las habilidades sociales, lo que podría ser índice de aislamiento y un posterior repercutiría en un Trastorno Adaptativo, en el que se verá alterada la vitalidad con la que enfrente la vida diaria. 
Finalmente, la responsabilidad e interés con que asume los deberes al interior de la prisión son índices de una anormal adaptación.

\section{Sujeto 6}

El ingreso a prisión puede causar en el interno altos grados de ansiedad que pueden afectar el entorno del interno, sus relaciones personales y la afectividad, y si además de esto se tiene una variable alta en consumo de sustancias, el interno tiene una alta probabilidad de estar mal adaptado al medio penitenciario. Si esta problemática se extiende por un tiempo aproximado de tres meses, se estarían cumpliendo los criterios para diagnosticar un Trastorno Adaptativo. En la segunda aplicación de la investigación, algunos de esos criterios se mantuvieron y algunos se generaron como resultado de una inadaptación a la llegada a la prisión, como lo son los resultados bajos en responsabilidad y una alta inestabilidad emocional que genera aislamiento y falta de energía para realizar sus actividades diarias.

\section{Sujeto 7}

El sujeto presenta niveles altos en cuanto a consumo de sustancias, ansiedad, estrés y depresión, lo cual puede desencadenar problemáticas a largo o mediano plazo. Su estabilidad emocional y sociabilidad están influenciadas por la falta de apoyo emocional, el sujeto no tiene las facultades necesarias para afrontar el contexto penitenciario, su originalidad es afectada por los niveles altos de depresión, no es capaz de tomar sus propias decisiones y hace caso omiso a las normas que exige el establecimiento carcelario. Su falta de energía se puede presentar, bien por sus niveles elevados de depresión o por el consumo de sustancias psicoactivas. No tiene buena adaptación al medio penitenciario y tiene una alta probabilidad de presentar alguna patología.

\section{Sujeto 8}

El sujeto 8 presenta un nivel elevado en cuanto a las variables de Prisionalización en los factores de apoyo social, apoyo social emocional, afectividad y habilidades de oposición, lo que podría ocasionar una inestabilidad emocional y pérdida de interés para realizar tareas del diario vivir,

E 74 
y una baja tolerancia a la frustración. Por otra parte, la misma falta de apoyo emocional desencadenaría un nivel de ansiedad elevada, en donde el sujeto perdería el interés por adquirir nuevos conocimientos y experiencias. El consumo de sustancias se ve inmerso en la falta de responsabilidad que presenta el mismo sujeto, llevándolo a generar una problemática más amplia en cada variable.

\section{Sujeto 9}

Relacionando el porcentaje alto de variables de Prisionalización que presenta el sujeto 9, se puede establecer cómo dichos efectos inciden en el desarrollo de un Trastorno Adaptativo por medio del nivel de consumo de sustancias, ansiedad, depresión, relaciones interpersonales, apoyo social, indiferencia a la norma, habilidades de oposición, habilidades de comunicación y apoyo social. Al analizar la posible presencia de Trastornos Adaptativos, se puede ver que el sujeto cuenta con antecedentes de depresión y habilidades sociales, lo cual hace que el individuo pierda la iniciativa para socializar en un grupo y presente falta de energía para realizar actividades cotidianas. En otra instancia, el sujeto al no comprometerse tanto con sus propias normas como con las del establecimiento, puede ocasionarle problemas de inestabilidad en las funciones que desempeñe. Todos estos factores desencadenarían un nivel elevado de consumo de sustancias psicoactivas.

\section{Sujeto 10}

La presencia de la responsabilidad, estabilidad emocional, cautela, originalidad y vigor, factores de Trastorno Adaptativo pueden llegar a ser desencadenados por causa de los efectos de Prisionalización con un puntaje alto. El estrés y la depresión son algunos de los factores predominantes en la Prisionalización que inciden en la estabilidad emocional, esto ocasiona baja tolerancia a la frustración y un ajuste de emocionalidad deteriorado. El no contar con adecuadas habilidades de oposición, hace que el sujeto realice actividades y tome decisiones apresuradas sin premeditar consecuencias. El consumo de sustancias es otro efecto que involucra el desarrollo de un TA en cuanto a la responsabilidad, ya que afecta horarios y altera esferas de tiempo y espacio. 


\section{Sujeto 11}

El sujeto 11 presenta una buena adaptación al medio penitenciario; sin embargo, su estabilidad emocional se encuentra afectada por la depresión y la falta de comunicación con su núcleo tanto familiar como de amistades. Otra de las causas por las que la estabilidad emocional se encuentra afectada es debido al posible consumo de sustancias psicoactivas y a la falta de recursos cognitivos para el afrontamiento en la prisión.

\section{Sujeto 12}

En el sujeto 12 se pueden evidenciar variables altas en efectos de Prisionalización como el consumo de sustancias, ansiedad, estrés y depresión, las cuales pueden desencadenar un nivel bajo de responsabilidad, en donde el sujeto pierde el interés por realizar las actividades de su diario vivir. Por lo anterior, el individuo perdería su originalidad en cuanto a relaciones personales. La indiferencia por la norma puede llegar a generarle problemas en su ascendencia, y el atribuirle sus responsabilidades al contexto desencadena problemas en sus relaciones personales lo que lo llevaría a un aislamiento. Por otro lado, el consumo de sustancias desequilibra su emocionalidad. Es un sujeto con un nivel de riesgo significativo.

\section{Sujeto 13}

La presencia de los efectos de Prisionalización como ansiedad, estrés y depresión pueden generar una baja responsabilidad, que lo llevaría a dejar de lado sus labores diarias y a perder el interés por las mismas. El consumo de sustancias generaría el descuido social en cuanto a pérdida de interacción social y autoestima baja, también podría desencadenar una rebeldía hacia la norma que perjudicaría su instancia en prisión. Otro puntaje alto en efectos de Prisionalización es el locus de control, que correlacionándolo con la estabilidad emocional del sujeto, desencadena que culpara al contexto o a otros por las acciones cometidas por él mismo.

\section{Sujeto 14}

El consumo de sustancias y la afectividad generan en el sujeto una baja responsabilidad y una baja estabilidad emocional, generándole pérdida de interés por adquirir nuevos conocimientos y por llevar a cabo las labores que sele son asignadas. El ingreso al contexto penitenciario 
genera que el individuo tenga una autoestima baja perdiendo también su originalidad debido al cambio de contexto tan radical. Finalmente, sus relaciones personales pueden verse tan afectadas, que podría llegar al punto del aislamiento.

\section{Sujeto 15}

El sujeto presenta, en efectos de Prisionalización, variables altas como consumo de sustancias y afectividad, las cuales pueden generar irresponsabilidad en cuanto a labores diarias. Su estabilidad emocional es afectada por la baja interacción con las personas, su baja sociabilidad es afectada, en parte por el consumo de sustancias, lo cual desencadena una posible presencia de trastornos de personalidad.

\section{Discusión}

El principal objetivo del estudio fue analizar la correlación existente entre los efectos de Prisionalización y el Trastorno Adaptativo, en donde según Echeverri (2010) la Prisionalización se da como consecuencia al cambio de contexto abierto a uno cerrado, lo cual genera en el interno un esfuerzo para adaptarse a ese medio y este esfuerzo genera cambios comportamentales y cognitivos, que sin el tratamiento adecuado, puede llegar a generar Trastornos de tipo Adaptativos.

Al analizar los resultados arrojados por los instrumentos utilizados, se logra observar una correlación en cuanto a la incidencia de los efectos de Prisionalización en el Trastorno Adaptativo, ya que los primeros meses de instancia en prisión se ven caracterizados por altos niveles de ansiedad y depresión, que generan alteraciones emocionales y falta de recursos cognitivos por parte del propio interno para afrontar el encarcelamiento, esto hace que el sujeto presente un pensamiento catastrófico, sentimientos de impotencia y la percepción de no poder superar la situación.

Esto se puede evidenciar en los altos porcentajes arrojados en las variables: ansiedad con un porcentaje del $66.6 \%$ presente en la población total, depresión con un $73.3 \%$, personalidad con un $66.6 \%$, afectividad con un $66.6 \%$, indiferencia a la norma con un $60 \%$, y riesgo psicopatológico con un $53.3 \%$. Todo esto relacionado con una variable determinante que dificulta aún más la adaptación del interno como lo es la del consumo de sustancias, con un $93.3 \%$ de la población total. 
Estas variables mantenidas en el tiempo son posibles desencadenantes de un Trastorno Adaptativo. Relacionando esto con el P-IPG, en que se evidencian puntuaciones altas que indican la relación de variables de efectos de Prisionalización incidentes en el desarrollo de un trastorno adaptativo.

En cuanto a las puntuaciones bajas que arroja el PIPG, como son responsabilidad, inestabilidad emocional, autoestima, vigor originalidad que se correlacionan con las variables altas arrojadas en el IVIC, se da respuesta a la hipótesis y objetivos planteados.

\section{Conclusión y Recomendaciones}

Para conocer esta problemática se recomienda un estudio más a fondo sobre la problemática de Prisionalización y Trastorno Adaptativo, ya que según el DSM IV-TR (2002), este trastorno es uno de los menos estudiados, esto se evidencia por la escasez de instrumentos diagnósticos. De igual forma, si se conduce esta variable psicológica al contexto penitenciario, se puede determinar su importancia, ya que el Trastorno Adaptativo es uno de los más comunes en las instituciones totales.

Finalmente, se recomienda, para futuros estudios, tomar una población más amplia y de diferentes establecimientos con el fin de obtener posibles variaciones en cuanto al desarrollo de un Trastorno Adaptativo. Similarmente, poder establecer diferencias entre delitos y entre géneros para determinar si existen diferencias o no con relación al Trastorno Adaptativo y los efectos de Prisionalización.

\section{Referencias}

Altamirano, F.; \& Cuevas, L. (15 de diciembre de 2012). Re: Implicancia de la Prisionalización en el Sistema Músculo Esquelético de las Personas Privadas de Libertad [mensaje en un blog]. Recuperado de http:// neurocienciacriticas.blogspot.com/2012/12/implicancia-de-la-prisionalizacion-en_15.html

Andreu, M. (2011). Un protocolo de tratamiento para los trastornos adaptativos, apoyado por un sistema de realidad virtual: Análisis desde su eficacia a través de un estudio controlado (Tesis doctoral). Recuperado

E 78 
de http://www.tdx.cat/bitstream/handle/10803/48514/sandreu.pdf;jsessionid=75F3E5C3811BD84C 86A92C4B969490C5.tdx1?sequence=1

American Psychiatric Association - APA. (2002). DSM-IV-TR. Manual diagnóstico y estadístico de los trastornos mentales. Texto revisado. Barcelona: Masson.

Baños, R.M.; Guillén, V.; Botella, C.; García, A.; Jorquera, M.; \& Quero, S. (2008). Un programa de tratamiento para los trastornos adaptativos. Un estudio de caso. Apuntes de Psicología, 26(2), 303-316. Recuperado de http://www.researchgate.net/profile/Azucena_Garcia-Palacios/publication/44294114_ Un_programa_de_tratamiento_para_los_trastornos_adaptativos._Un_estudio_de_caso/ links/0c960525eda46865c5000000.pdf.

Carreño, L. (2013). Síndrome de Prisionalización. Expresión Forense, 1(2), 5. Recuperado de http://expresionforense.com/docs/expresion\%20forense_no\%202_mayo_2013_high.pdf

CIE-10 (1992). Trastornos mentales y del comportamiento. Madrid: Meditor.

Colombiana de Salud, (2009). Guías de manejo Trastorno de adaptación psiquiátrica. Recuperado de http://www. colombianadesalud.org.co/GUIAS_MEDICINA_ESPECIALIZADA/PSIQUIATRIA/TRASTORNO\%20DE\%20 ADAPTACION.pdf

Díaz, F. (2011). Psicología y ley. Psicología jurídica forense, criminológica y victimología. Bogotá: PSICOM LTDA.

Echeverri - Vera, J. (2010). La prisionalización, sus efectos psicológicos y su evaluación. Pensando Psicología, 6(11), 157-166. Recuperado de http://revistas.ucc.edu.co/index.php/pe/article/view/375

Escaff-Silva, E.; Feliú-Vergara, M.P.; Estévez-Merello, M.; \& Torrealba-Henríquez, C. (2013). Consecuencias psicosociales de la privación de la libertad en imputados inocentes. Revista Criminalidad, 55(3), 291-308. Recuperado de http://www.policia.gov.co/imagenes_ponal/dijin/revista_criminalidad/ vol55_3/55308consecuencias.html

Fernández, P. (2008). Evaluación e intervención psicológica en un caso de trastorno adaptativo mixto en una mujer con Arnold-Chiari tipo I. Norte de Salud Mental, 7(31), 99-104. Recuperado de http://revistanorte.es/ index.php/revista/article/view/548 
Gómez, M.E.; Aldana, E.; Carreño, J.; \& Sánchez, C. (2006). Alteraciones Psicológicas en la Mujer Embarazada. Psicología Iberoamericana, 14(2), 28-35. Recuperado de http://www.redalyc.org/pdf/1339/133920321004.pdf

Gonzales, C. (Abril, 2010). Anexos penitenciarios en Colombia. Recuperado de http://psicologiajuridica.org/archives $/ 265$

Gordon, L. V. (1991). Perfil - inventario de la personalidad (P-IPG). Madrid: TEA

Gutiérrez, C. (2010). Revisión sobre la definición de psicología jurídica. Perspectiva psicológica, 6(2), 221-235. Recuperado de http://www.usta.edu.co/otraspaginas/diversitas/doc_pdf/diversitas_10/vol.6no.2/ articulo_2.pdf

Herrero, V., \& Cano, A. (2010). Un caso de trastorno adaptativo con ansiedad: evaluación, tratamiento y seguimiento. Anuario de Psicología Clínica y de la Salud, 6, 53-59. Recuperado de http://institucional.us.es/ apcs/doc/APCS_6_esp_53-59.pdf

Joya, Y., \& Quintero, X. (2011). El fomento de espacios socioeducativos en el ámbito penitenciario a través del modelo de competencias ciudadanas: Una estrategia de intervención desde Trabajo social (Tesis de pregrado). Recuperada de http://repositorio.uis.edu.co/jspui/bitstream/123456789/9472/2/142351.pdf

Martínez, M. (2014). Principales consecuencias que enfrentan las mujeres sujetas a prisión preventiva, en el municipio de Huehuetenango (Tesis de pregrado). Recuperada de http://biblio3.url.edu.gt/Tesario/2014/07/03/Martinez-Marilyn.pdf

Medina, L.; Osorno, A.; Reyes, L.; Ríos, M.; \& Jiménez, O. (2014). Diseño de un instrumento que permita establecer indicadores de efectos de Prisionalización en miembros del cuerpo de custodia y vigilancia del INPEC de Bogotá (tesis de pregrado). Recuperada de http://repository.ucatolica.edu.co:8080/jspui/ handle/10983/1998

Mena, L., \& Rueda, M. (2012). Privatización de las cárceles colombianas (trabajo de pregrado): Recuperado de http://repository.unimilitar.edu.co/bitstream/10654/9201/1/MenaLuzStella2012.pdf

Mingote, J.; Gálvez, M.; Cuadrado, P.; \& Gutiérrez, M.D. (2009). El paciente que padece un trastorno depresivo en el trabajo. Medicina y Seguridad del Trabajo, 55(214), 41-63. Recuperado de http://scielo.isciii.es/pdf/ mesetra/v55n214/original2.pdf

$\boldsymbol{\epsilon} \mid 80$ 
Moles, M.; et.al. (2012). Desarrollo de una entrevista diagnóstica para los trastornos adaptativos. fòrum de recerca, 16, 1049-1069. Recuperado de http://repositori.uji.es/xmlui/bitstream/handle/10234/77430/ fr_2011_10_12.pdf?sequence=1

Morales, L. y García, E. (2010). Psicología jurídica quehacer y desarrollo. Diversitas Perspectiva Psicológica, 6(2), 237-256. Recuperado de http://www.usta.edu.co/otraspaginas/diversitas/doc_pdf/diversitas_10/ vol.6no.2/articulo_3.pdf

Murillo, A.; Salcedo, C.; \& Bohórquez, A. (2009). Descripción de la conducta suicida en miembros de las fuerzas militares de Colombia. Protocolo de investigación (tesis de pregrado). Recuperada de http://repository. unimilitar.edu.co/bitstream/10654/10344/2/MurilloMosqueraAnaXimena2009.pdf

Ojeda, E. (2010). Plan de acción elaborado con las pacientes de recién diagnóstico de cáncer de mama y trastornos adaptativos tipo ansioso y/o deprimido. Consulta de Oncología médica. Hospital Universitario "Dr. Luis Gómez López" Barquisimeto Estado Lara (Tesis de especialista). Recuperado de http://bibmed.ucla. edu.ve/DB/bmucla/edocs/textocompleto/TWP870.DV40442010.pdf

Pérez-Fernández, E., \& Redondo-Illescas, S. (1991). Efectos Psicológicos de la estancia en prisión. Papeles del Psicólogo, 48. Recuperado de http://www.papelesdelpsicologo.es/vernumero. $a s p ? i d=485$

Uribe, A.; Martínez, M.; \& López, K. (2012). Depresión y Ansiedad Estado/Rasgo en Internos Adscritos al "Programa de Inducción al Tratamiento Penitenciario" En Bucaramanga, Colombia. Criminalidad, 54(2), 47-60. Recuperado de http://dialnet.unirioja.es/servlet/articulo?codigo=4190815

Ruiz, J. y Cols. (2008). Un Estudio Longitudinal y Comparativo sobre la Adaptación Psicosocial a Contextos Carcelarios. Anuario de Psicología Jurídica, 18, 61-72. Recuperado de http://www.redalyc.org/ pdf/3150/315024785007.pdf

Hernández, R.; Fernández, C., \& Baptista, P. (2006). Metodología de la investigación. Mexico, D.F. McGraw-Hill.

Soria, M.; et.al. (2009). El ejercicio profesional del psicólogo y su relación con el sistema judicial en Cataluña. Papeles del Psicólogo, 30(2), 164-168. Recuperado de http://www.papelesdelpsicologo.es/pdf/1705.pdf

Tapias, A. y Cols. (2007). Descripción de las estadísticas de problemáticas psicosociales en guardianes penitenciarios de Colombia. Suma psicológica, vol. 14 (1). PP. 7-22. Recuperado de file:///C:/Users/jjtb/Downloads/Dialnet-DescripcionDeLasEstadisticasDeProblematicasPsicoso-2530316\%20(1).pdf 
Tamara, M. y Cols. (2011). Caracterización y Perforación Criminológica y Penitenciaria de la Población Condenada y Privada de la Libertad en Los Establecimientos de Reclusión del INPEC y Propuesta para el Direccionamiento del Tratamiento Penitenciario en Colombia. Revista INPEC, 20-21. Recuperado de http:// www.inpec.gov.co/portal/pls/portal/!PORTAL.wwpob_page.show?_docname=2893 094.PDF

Universidad Pontificia Bolivariana (2009). Diseño, Validación e implementación de Instrumentos científicos para el proceso de valoración, clasificación y seguimiento en el tratamiento penitenciario de la población condenada en los establecimientos de reclusión de orden nacional. Colombia: PUBLICOM PDC

Valdez, H. (2009). Detección y tratamiento del Síndrome de Burnout en el sistema penitenciario de Jalisco. Waxapa, 1(1), 24-34. Recuperado de http://www.medigraphic.com/pdfs/waxapa/wax-2009/wax091e.pdf

Zarza, M.; \& Sobrino, M.I. (2007). Estrés de adaptación sociocultural en inmigrantes latinoamericanos residentes en Estados Unidos vs. España: Una revisión bibliográfica. Anales de Psicología, 23(1), 72-84. Recuperado de http://www.um.es/analesps/v23/v23_1/10-23_1.pdf

Zeas, I.; Coello, A.M.; De las Torres, D.; e Iglesias, P. (2012). Trastornos adaptativos en pacientes con acné en consulta externa de dermatología del Hospital Vicente Corral Moscoso, Cuenca (Tesis de pregrado). Recuperada de http://dspace.ucuenca.edu.ec/bitstream/123456789/3533/1/MED133pdf

\section{Citación}

Cajamarca Sarmiento, J.; Triana Barrios, J.; \& Jiménez-Jiménez, W. A. (2015). Los efectos de Prisionalización y su relación con el Trastorno Adaptativo. Enfoques, 1(2),

Recibido: 09 / 03 / 2015 | Aceptado: 14 / 04 / 2015.

$\boldsymbol{E} \mid 82$ 